Mathematical Modelling and Analysis, vol. 3, pp. 81-85

R.Čiegis (Ed)

(C) 1998 Technika

\title{
THE DOUBLE-PERIODIC ANALOG OF THE CAUCHY KERNEL AND SOME ITS APPLICATIONS
}

\author{
N.A.DZEHTSIARENKA, E.I.ZVEROVICH \\ Belorussian State University \\ Skariny av.,4, 220050, Minsk, Belarus.
}

\begin{abstract}
A new analytic expression for the double-periodic analog of the Cauchy kernel is proposed and its explicit form is presented. Some applications of this analog are given.
\end{abstract}

\section{INTRODUCTION}

Many articles (see e.g. [1-7]) are devoted to investigation of conjugation and boundary problems for double-periodic piecewise-analytic functions. The Weierstrass zeta-function $\zeta(\tau-z)$ or some its modifications are taken there as an analog of the Cauchy kernel $\frac{1}{\tau-z}$. However, they are not double-periodic (but are "quasiperiodic" [7]).

The fact of the existance of meromorfic analog of the Cauchy kernel on Riemann surface (in particular, double-periodic kernel) is established in [8], where its explicit expression through basic functionals of a Riemann surface is given. Using this fact and the theory of elliptic functions [9], we propose a new analytic expression for double-periodic analog of the Cauchy kernel in the explicit form. We apply this analog to solve a singular integral equation and an analytic continuation problem.

\section{FORMULATION OF THE DOUBLE-PERIODIC ANALOG OF THE CAUCHY KERNEL}

Let us consider double-periodic elliptic functions with the basic periods $\omega_{1}, \omega_{2}$, where $\operatorname{Im} \frac{\omega_{2}}{\omega_{1}}>0$. The domain of each double-periodic function $f: C /\left(Z \omega_{1}+\right.$ $\left.Z \omega_{2}\right) \longrightarrow \hat{C}$ is homeomorphic to torus, therefore the theory of elliptic functions sometimes is called the theory of functions on torus [11, p.42]. One 
can present this domain as the surface of the standart torus, or as the factor - manifold of the plane with respect to the lattice $Z \omega_{1}+Z \omega_{2}$, or as the closed parallelogram on vectors $\omega_{1}, \omega_{2}$ with the common origin which opposite sides are identified by the rule $t \longmapsto t \pm \omega_{1}$ and $t \longmapsto t \pm \omega_{2}$, or as so-called "fundamental parallelogram". The last term means the parallelogram $\Pi$ on vectors $\omega_{1}, \omega_{2}$ with the common origin contains all internal points, two sides (that is vectors $\omega_{1}, \omega_{2}$ ) and the only one vertex of the parallelogram (namely, the common origin of vectors $\left.\omega_{1}, \omega_{2}\right)$. It is convenient to use the fundamental parallelogram $\Pi$ because it is bijective to the Riemann surface $C /\left(Z \omega_{1}+Z \omega_{2}\right)$.

Let, for example, two points $a, b \in C$ are congruent up to the modulus of periods $\omega_{1}, \omega_{2}$, i.e. $a \equiv b$. It means, by definition, that $(a-b) \in Z \omega_{1}+Z \omega_{2}$. If $a, b \in \Pi$, then it leads immediately to equality $a=b$.

In the theory of elliptic functions the question of existence of elliptic function $f(z)$ with zeros in points $a_{1}, \ldots, a_{n}$ and poles in points $b_{1}, \ldots, b_{n}$ was solved completely (see e.g. [9]), where each zero and each pole is written down successivly a number of times equal to its multiplicity and any other zeros and poles function $f(z)$ does not have. The existence of such function is equivalent to fulfilment of the folloving congruence relation

$$
\sum_{k=1}^{n} a_{k} \equiv \sum_{k=1}^{n} b_{k} \text { by the module of the periods } \omega_{1}, \omega_{2} \text {. }
$$

If this condition holds, the function $f(z)$ is written down in the explicit form (2)

$$
f(z)=C \frac{\prod_{k=1}^{n} \sigma\left(z-a_{k}\right)}{\prod_{k=1}^{n} \sigma\left(z-b_{k}\right)}
$$

where $C \neq 0$ and $\sigma(z)-\sigma$ - function of Weierstrass, corresponded to the lattice $Z \omega_{1}+Z \omega_{2}$.

Using this result we construct a double - periodic analog of the Cauchy kernel with the minimal characteristic divisor $\Delta . \Delta$ is a set of points together with their multiplicities. The order of the divisor $\Delta$ is defined as the sum of multiplicities of all its points. It is denoted $\operatorname{ord} \Delta$. A divisor $\Delta^{-1}$ is called minimal divisor if there is no elliptic function divisible by the divisor $\Delta^{-1}$ and no elliptic differential divisible by the divisor $\Delta$. It follows from Riemann - Roch theorem [11], that for minimal divisor $\operatorname{ord} \Delta=0$ takes place. The expression $A(z, \tau) d \tau$ will be called the double - periodic analog of the Cauchy kernel, if $A(z, \tau)$ is elliptic function of one variable by the fixed another, and satisfies the following asymptotic relation

$$
A(z, \tau) \sim \frac{1}{\tau-z} \text { if } z \rightarrow \tau .
$$

Due to this asymptotic the local properties of the Cauchy type integral with 
the kernel $A(z, \tau) d \tau$ are the same as of that with the kernel $\frac{d \tau}{\tau-z}$. The minimal divisor $\Delta$ is called characteristic divisor of the kernel $A(z, \tau) d \tau$, if this kernel is divisible by divisor $z^{-1} \Delta$ with respect to $\tau$, and by divisor $\tau^{-1} \Delta^{-1}$ with respect to $z$. Let us write down minimal divisor $\Delta$ in terms of its points in following irreducible form

$$
\Delta=\frac{a_{1} \cdot \ldots \cdot a_{n}}{b_{1} \cdot \ldots \cdot b_{n}}
$$

where each zero and each pole is written down successivly a number of times equal to its multiplicity. The minimality property means, that there is no function at the form (2), which corresponds to this divisor, that is

$$
\sum_{k=1}^{n} a_{k} \not \equiv \sum_{k=1}^{n} b_{k} \text { by the module of the periods } \omega_{1}, \omega_{2} \text {. }
$$

Taking it into account and also that the function $\sigma(z)$ is odd, $\sigma(0)=$ $0, \sigma^{\prime}(z)=1$, it is easy now to write out expression for double - periodic analog of the Cauchy kernel with minimal characteristic divisor (4):

$$
A(z, \tau) d \tau=\frac{\sigma\left(\tau-z+\sum_{k=1}^{n}\left(a_{k}-b_{k}\right)\right)}{\sigma\left(\sum_{k=1}^{n}\left(a_{k}-b_{k}\right)\right)} \prod_{k=1}^{n} \frac{\sigma\left(z-b_{k}\right) \sigma\left(\tau-a_{k}\right)}{\sigma\left(\tau-b_{k}\right) \sigma\left(z-a_{k}\right)} \frac{d \tau}{\sigma(\tau-z)}
$$

Let us study its properties. For (5) we have: $\sigma\left(\sum_{k=1}^{n}\left(a_{k}-b_{k}\right)\right) \neq 0$. Besides first two multipliers in (6) tend to unit and $\sigma(\tau-z) \sim \tau-z$, if $z \rightarrow \tau$. Therefore the asymptotic (3) holds for the kernel (6). Further the expression (6) with the fixed $\tau$ has zeros at points

$$
\tau+\sum_{k=1}^{n}\left(a_{k}-b_{k}\right), \quad b_{1}, \ldots, b_{n}
$$

and poles at points

$$
a_{1}, \ldots, a_{n}, \tau \text {. }
$$

It is obvious, that the sum of all numbers (7) is equal to the sum of all numbers (8). So, the condition (1) takes place, that is expression (6) is double - periodic function with respect to $z$, which is besides divisible by divisor $\tau^{-1} \Delta^{-1}$. It is similarly possible to show, that (6) is double - periodic differential with respect to $\tau$, which is besides divisible by divisor $z^{-1} \Delta$. 


\section{THE SINGULIAR INTEGRAL EQUATION}

Let consider the singular integral equation of the first type

$$
\frac{1}{\pi i} \int_{L} A(t, \tau) \phi(\tau) d \tau=f(t), \quad t \in L
$$

with the kernel (6), assuming, that $f(t)$ is a H-continuous function and $\mathrm{L}$ is a smooth closed curve, which support lays in fundamental parallelogram and does not pass through points of divisor $\Delta$. Introducing the piecewise analytic double - periodic function

$$
\Phi(z)=\frac{1}{2 \pi i} \int_{L} A(z, \tau) \phi(\tau) d \tau
$$

and using the Sochotsky formula [10]

$$
\begin{gathered}
\phi(t)=\Phi^{+}(t)-\Phi^{-}(t), \quad t \in L, \\
\frac{1}{\pi i} \int_{L} A(t, \tau) \phi(\tau) d \tau=\Phi^{+}(t)+\Phi^{-}(t), \quad t \in L,
\end{gathered}
$$

we reduce the equation (9) to the linear conjugation problem equivalent to it

$$
\Phi^{+}(t)+\Phi^{-}(t)=f(t), \quad \Delta^{-1} \mid(\Phi), t \in L .
$$

Applying to it the results from [8], we conclude the following.

If the set $\Pi \backslash L$ is not coherent, then the integral operator (9) is involutory, that is the equation (9) has the unique solution for any function $f(t)$ and this solution is given by the formula

$$
\phi(t)=\frac{1}{\pi i} \int_{L} A(t, \tau) f(\tau) d \tau, \quad t \in L .
$$

When the set $\Pi \backslash L$ is coherent, two cases are possible, depending on, whether the homogeneous problem (13) has non- trivial solution or not. In the last case the operator (9) is invertible, though it is not involutory. In the first case the solution of the equation (9) exists if one linear condition is fulfilled and it depends on one arbitrary constant.

In all cases the solution of the equation (9) can be obtained in the explicit form. 


\section{AN ANALYTIC CONTINUATION PROBLEM}

We shall assume, that the smooth closed curve $L$, oriented in a direction counter-clockwise, lays strictly inside the parallelogram of the periods and breaks it into two domains $D^{+}$and $D^{-}$. Let's find conditions, at which the given $\mathrm{H}$-continuous function $\phi: L \longrightarrow C$ admits analytic continuation in domain $D^{-}$up to double - periodic function with the given basic periods. Such problem on the plane was considered in [10]. For solving this problem we take the double - periodic analog of the Cauchy kernel with characteristic divisor, all points of which lay in $D^{+}$. Then analytic extendability is equivalent to the representing function, continued in $D^{-}$by the integral of Cauchy type

$$
\phi(z)=-\frac{1}{2 \pi i} \int_{L} \phi(\tau) A(z, \tau) d \tau .
$$

By the formulas of Sochotsky the boundary value of this function on $L$ from the side of domain $D^{-}$is equal

$$
\phi(t)=\frac{1}{2} \phi(t)-\frac{1}{2 \pi i} \int_{L} \phi(\tau) A(t, \tau) d \tau
$$

So we can formulate the following criterion.

Analytic extendability of a function $\phi(t)$ to domain $D^{-}$up to double - periodic function is equivalent to the condition

$$
\phi(t)+\frac{1}{\pi i} \int_{L} \phi(\tau) A(t, \tau) d \tau=0, \text { for all } t \in L
$$

\section{REFERENCES}

[1] Gerasimov I.A.Functions of Weierstrass and their application in the mechanics and astronomy. Moscow (1990).

[2] Zhorovina T.N.The generalized Riemann problem with constant coeffisients on torus. Vestn. BSU, 1, No. 1 (1985), P. 64-67.

[3] Chibrikova L.I.About boundary problems for a rectangular. Kazan, (1964).

[4] Chibrikova L.I. The basic boundary problems for analytic functions. Kazan, KSU, (1977).

[5] Chibrikova L.I., Pokazeev V.V.About one generalization of double-periodic functions. TFCV and boundary problems. Cheboks., (1982), P. 112-140.

[6] Koiter W.T.Some general theorems on doubly -periodic and quasiperiodic functions. Proc. Kon. Ned. Akad. Wet., 1959, Ser. A, 62, No. 2 (1959) P. 120-128.

[7] Aksentieva E.P.Functions of Weierstrass in boundary problems., Kazan, KSU, (1994).

[8] Zverovich E.I.// Uspekhi math. nauk, VINITI, 26, issue 1 (1971), P. 113-179.

[9] Akhiezer N.I.Elements of the theory of elliptic functions. Nauka, Moscow, (1970).

[10] Gakhov F.D.Boundary problems. Nauka, Moscow, 1977.

[11] Springer G.Introduction to the theory of Riemann surfaces. Mir, Moscow, (1960). 